\title{
Cribado e intervención breve digital en el uso problemático de Internet: Programa piensaTIC
}

\section{Electronic screening and brief intervention in the problematic Internet use: PiensaTIC Program}

\author{
Juan Díaz Salabert y María Isabel Gómez Torres \\ Asociación para la prevención de adicciones en adolescentes y jóvenes, ADIADOS. Málaga, España
}

\begin{abstract}
Resumen
La integración del cribado, la intervención breve y la derivación al tratamiento es una estrategia preventiva y asistencial que ha demostrado eficacia en la mejora de la salud de los consumidores de alcohol y otras drogas. Su versión digital ha demostrado una mayor facilidad para su extensión y aceptación entre los adolescentes y jóvenes. Debido a la necesidad de desarrollar actuaciones eficientes para la prevención y reducción de riesgo del uso problemático de internet en la población escolar, se propone en este trabajo la adaptación de esta metodología para su utilización en la prevención de este problema en dicha población. El Programa PiensaTIC es un programa de cribado e intervención breve administrado vía web que se ha desarrollado de forma piloto con estudiantes de enseñanzas secundarias en la ciudad de Málaga. Se describen en este artículo las características de este programa preventivo y sus resultados respecto al conocimiento de las diferentes formas de uso de internet por parte de los adolescentes estudiados. En este estudio se encuentran diferencias entre los tipos de conductas online más frecuentes entre alumnos y alumnas así como prevalencia y características diferenciales, según género, del uso problemático de internet.
\end{abstract}

Palabras clave: Cribado digital, intervención breve, uso problemático de internet.

\begin{abstract}
The integration of screening, brief interventions, and referral to treatment is a preventive and care strategy that has demonstrated effectiveness in improving the health of alcohol and other drug users. The implementation of this strategy in its digital form has facilitated its spread and acceptance among adolescents and young people. The objective of this study was to adapt this methodology in order to develop efficient actions to prevent and reduce the risk of problem internet use among the school population. The PiensaTIC Program is a web-based screening and brief intervention pilot application that has been administered to secondary school students in the city of Malaga (Spain). This article describes the features of this preventive program and its results regarding the different forms of internet usage by the adolescents studied. Differences were found between male and female students in relation to the most common types of online behavior, as well as differences in prevalence and the differential characteristics of problem internet use by gender.
\end{abstract}

Keywords: Electronic screening, brief intervention, problematic internet use.

Cómo citar este artículo: Díaz, J. y Gómez-Torres, M. I. (2019). Cribado e Intervención Breve Digital en el Uso Problemático de Internet: Programa piensaTIC. Escritos de Psicología, 12, 57-68.

Correspondencia: Juan Díaz Salabert. Asociación para la Prevención de Adicciones en Adolescentes y Jóvenes ADIADOS. C/ Mendoza, 25. 29002 Málaga. E-mail: juan.diazsal@gmail.com. E-mail de la coautora María Isabel Gómez Torres: maigotomalaga@gmail.com. 


\section{Introducción}

El cribado, la intervención breve y la derivación al tratamiento (SBIRT por las siglas en inglés de Screening, Brief Intervention, and Referral to Treatment) es un enfoque integral desarrollado para la prestación de servicios de intervención temprana y la detección de las personas con trastornos por consumo de sustancias o en riesgo de padecerlos (Babor, McRee, Kassebaum, Grimaldi, Ahmed y Bray, 2007). Es una estrategia que se puede aplicar de manera flexible en diferentes entornos de atención clínica (Derges, Kidger, Fox, Campbell, Kaner y Hickman, 2017). Se ha adaptado para su uso en servicios de emergencia hospitalarios (Barata et al., 2017), (Landy, Davey, Quintero, Pecora, y McShane, 2016), centros de atención primaria (Hargraves et al., 2017) y otros entornos comunitarios, brindando oportunidades de intervención precoz con usuarios de sustancias en riesgo antes de que ocurran consecuencias más graves (Borus, Parhami y Levy, 2016).

En la práctica consta de tres etapas: El cribado o detección de sujetos en riesgo mediante pruebas cortas y validadas, la intervención motivacional breve y la adecuada derivación al tratamiento cuando proceda. Esta estrategia de evaluación e intervención se desarrolló inicialmente en los años noventa del pasado siglo para los trastornos por consumo de alcohol (Dimeff, Baer, Kivlahan y Marlatt, 1999), pero su uso se ha ampliado para incluir el uso de otras sustancias (Harris, Louis-Jacques y Knight, 2014), (Wright, 2017) acumulando sobrada evidencia de que produce mejoras a corto plazo en la salud de los consumidores de alcohol y otras drogas. Aunque todavía no se han demostrado los efectos a largo plazo en la salud de la población, los modelos de simulación sugieren que los beneficios podrían ser sustanciales (Babor et al., 2007).

Coincidiendo con el creciente interés por el desarrollo de la "e-Salud" (eHealth en inglés), término con el que se define al conjunto de tecnologías de la información y la comunicación que se emplean en el entorno sanitario, se han desarrollado métodos alternativos para la aplicación de elementos clave del cribado y la intervención breve mediante dispositivos digitales. Estos procedimientos son conocidos como cribado e intervención breve digital (Electronic Screening and Brief Intervention o e-SBI en inglés).

Este formato digital para la detección e intervención en las conductas adictivas ha sido especialmente impulsado por su idoneidad para la intervención con adolescentes y jóvenes (Fraeyman, Van Royen, Vriesacker, De Mey y Van Hal, 2012). Existe evidencia de que los adolescentes perciben que los cuestionarios sobre el uso de sustancias administrados por ordenador son más confidenciales que los formatos de lápiz y papel o las entrevistas presenciales y, por tanto, proporcionan respuestas más válidas y precisas en cribados y pruebas de evaluación administrados mediante dispositivos electrónicos. (Pedersen, Grow, Duncan, Neighbours, y Larimer, 2012). Se han estudiado los elementos clave que facilitan la aceptación de estas actuaciones entre los jóvenes: la facilidad de acceso, la corta duración, el lenguaje claro y sin prejuicios, los comentarios normativos personalizados y los enlaces a los servicios apropiados (Hallett, Maycock, Kypri, Howat y McManus, 2009).

Aunque la gran mayoría de las intervenciones de este tipo se han dirigido a jóvenes con riesgo de uso abusivo de alcohol (Cronce, Bittinger, Liu y Kilmer, 2014), también se han desarrollado para su aplicación en el consumo de cannabis (Walton et al., 2013) y otras drogas (Boumparis, Karyotaki, Schaub, Cuijpers y Riper, 2017).

El cribado e intervención breve digital implica, como mínimo, la detección de individuos con riesgo de desarrollar conductas adictivas y una intervención breve que proporciona comentarios personalizados sobre los riesgos y las consecuencias de su comportamiento. Esta intervención mínima puede incluir otros elementos comunes a la intervención breve tradicional como el feedback o retroalimentación motivacional. La retroalimentación motivacional básica incluye consejos generales sobre cómo reducir el consumo excesivo de alcohol. La retroalimentación personalizada proporciona mensajes más individualizados según la disposición del sujeto para cambiar y sus objetivos personales. Otro elemento posible es la retroalimentación normativa, que compara el consumo de alcohol de un individuo con el de su grupo de referencia. Los resultados de algunos estudios (Bewick et al., 2010) sugieren que la integración de la retroalimentación normativa sobre el consumo de alcohol entre pares es un enfoque prometedor en las intervenciones enfocadas en adolescentes.

La Figura 1 ilustra el modelo conceptual desarrollado por Tansil et al., (2016a) para indicar las vías causales a través de las cuales se espera que el cribado y la intervención breve digital reduzca el consumo excesivo de alcohol y los daños relacionados. Este modelo postula que la implementación de esta metodología aumentará el número de personas examinadas por su conducta de riesgo y que aquellos que superen los puntos de corte establecidos en el programa recibirán una intervención breve. Esto puede conducir a una mayor conciencia de las consecuencias nocivas de su conducta y puede motivar a estas personas a modificar sus patrones conductuales hacia una disminución de los daños relacionados con sus comportamientos adictivos. 
Figura 1

Modelo conceptual del funcionamiento del Cribado e intervención breve online

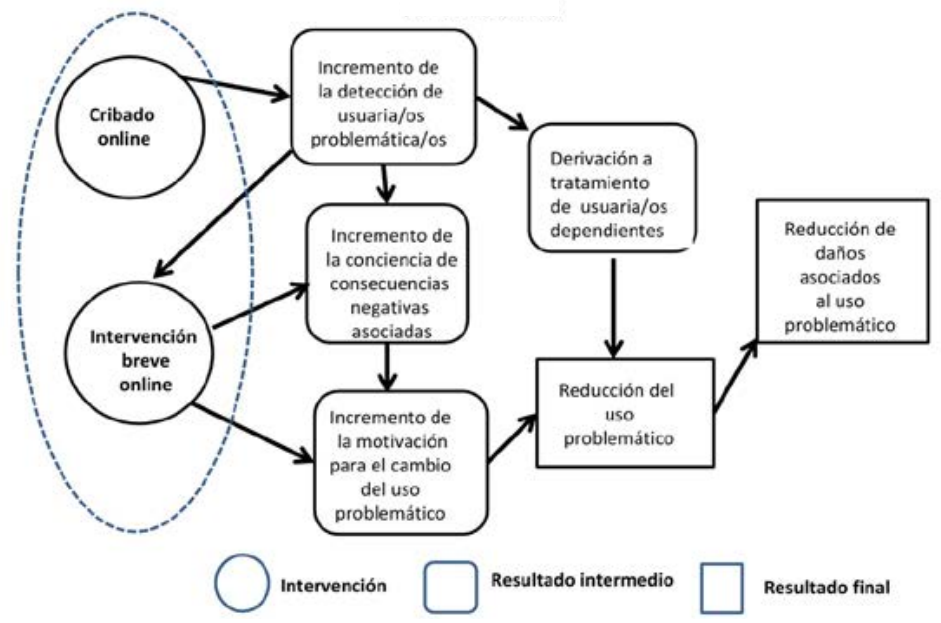

Adaptado de Alcohol Electronic and Brief Intervention: A Community Guide Systematic Review. (Tansil et al, 2016)

Diversos estudios han demostrado reducciones significativas en el consumo semanal de alcohol entre las condiciones de intervención y de control, lo que sustenta que es una intervención efectiva. (Donoghue, Patton, Phillips, Deluca y Drummond, 2014). La Community Preventive Services Task Force que asesora al Departamento de Salud y Servicios Humanos del gobierno de EE. UU. recomienda el cribado y la intervención breve digital para reducir el consumo excesivo de alcohol y los problemas relacionados basándose en las revisiones y metaanálisis que han realizado (Tansil et al., 2016b).

La intervención digital puede ser superior a la realizada en persona para usuarios con bajos niveles de gravedad del problema (Baumann, Gaertner, Haberecht, Bischof, John y Freyer-Adam, 2018). Es, por tanto, un enfoque especialmente idóneo para las actuaciones dirigidas a la prevención de comportamientos adictivos antes de que estén fuertemente arraigados. La evidencia actual sugiere que tanto la prevención universal como la selectiva basadas en intervenciones breves online sobre el consumo abusivo de alcohol con adolescentes pueden dar lugar a cambios clínicamente importantes en entornos educativos (Ganz, Braun, Laging, Schermelleh-Engel, Michalak y Heidenreich, 2018), de atención primaria (Kypri, Langley, Saunders, Cashell-Smith y Herbison, 2008) y hospitalarios (Duroy, Boutron, Baron, Ravaud, Estellat y Lejoyeux, 2016).

De forma paralela al desarrollo de estos avances en la prevención de los riesgos y daños asociados al consumo de alcohol y otras drogas, en la última década ha surgido una creciente preocupación social por las consecuencias negativas que pueden sufrir las personas con un uso abusivo de su conexión a internet, por lo que se han generado un gran número de estudios sobre la etiología, características y métodos de evaluación de estos tipos de comportamientos disfuncionales asociados al uso de las tecnologías de la información y la comunicación.

Tanto las definiciones como los criterios de diagnóstico y las herramientas de evaluación desarrolladas, enfatizan las similitudes entre estas conductas y los comportamientos adictivos con los que comparten síntomas como: a) usar Internet por más tiempo de lo previsto, b) preocupación por Internet, c) síntomas de abstinencia cuando no se puede acceder a Internet, d) intentos fallidos de detener o reducir el uso de Internet, e) pérdida de interés en pasatiempos o actividades que no sean Internet, f) uso excesivo de Internet a pesar del conocimiento de problemas relacionados, g) uso de Internet para escapar o aliviar estados de ánimo negativo, y h) mentir sobre el uso de Internet. (Li, O’Brien, Snyder y Howard, 2016).

La literatura sobre adicción a Internet refleja dos escuelas de pensamiento: aquellos autores que creen que la adicción a Internet merece ser clasificada como un trastorno psiquiátrico nuevo o emergente por derecho propio, y aquellos que plantean que existe un uso problemático de Internet en relación con actividades específicas on-line, como el juego de azar, el uso de las redes sociales, los videojuegos o la pornografía. A pesar de la falta de investigación metodológicamente sólida, la evidencia parece apoyar la segunda perspectiva (Yellowlees y Marks, 2007).

Además del concepto de adicción a Internet (Goldberg, 1996), existe una amplia utilización de los siguientes términos: uso problemático de Internet (Shapira et al., 2003), uso patológico de Internet (Morahan-Martin, 2004) y uso compulsivo de Internet (Greenfield, 1999). Consideramos que el termino más genérico de los propuestos es el Uso Problemático de Internet (UPI), que puede conceptualizarse 
como la incapacidad de controlar el uso de Internet que tiene consecuencias negativas en la vida diaria (Spada, 2014).

Los desacuerdos en los criterios de diagnóstico y la falta de grandes estudios epidemiológicos han ocasionado dificultades para establecer la prevalencia del UPI en la población general. Existe una impresión generalizada de que el problema está especialmente extendido entre la población adolescente. Un amplio estudio realizado en siete países (Tsitsika et al., 2014) mostró que un 13,9 \% de los escolares europeos presentaba Conducta Disfuncional en Internet definida por una puntuación en el Internet Addiction Test (IAT) (Young, 1998a) mayor de 40 puntos y un patrón de comportamiento caracterizado por la pérdida de control sobre el uso de Internet que potencialmente conduce al aislamiento y al descuido de las relaciones sociales, de las actividades académicas, de las actividades recreativas, de la salud y de la higiene personal. España (con un 22,8\% de la población estudiada) presenta en este estudio la mayor prevalencia de este uso problemático de internet entre los adolescentes de todos los países participantes.

En otro estudio de ámbito europeo realizado por Secades-Villa et al., (2014), se encontró una relación estadísticamente significativa entre la duración del uso de Internet y la frecuencia de uso de alcohol, tabaco, cannabis y otras drogas ilegales. La duración del uso de Internet también se asoció de forma significativa con problemas escolares, el uso de las máquinas tragaperras y otros problemas psicosociales. Se ha encontrado una asociación significativa del UPI con problemas de salud (migrañas, dolor lumbar, sobrepeso u obesidad, descanso insuficiente), psicológicos (riesgo de trastornos de la conducta alimentaria, riesgo de trastorno mental, depresión) y problemas sociales y familiares (Fernández et al., 2015). Existe alguna evidencia de que el trastorno de adicción a Internet podría verse asociado a disfuncionalidades de tipo neurológico como reducciones en las vías de la materia blanca (Lin et al., 2012) y disminución de la conectividad funcional en circuitos cortico-estriatales (Hong et al., 2013).

En nuestro país los estudios más recientes confirman los resultados de Tsitsika et al. (2014), presentando una incidencia de adolescentes usuarios problemáticos o en alto riesgo cercano a un $20 \%$. Así se observa en el estudio realizado por Gómez, Rial, Braña, Varela y Barreiro (2014) que encuentran un $19,9 \%$ de usuarios problemáticos entre estudiantes de Educación Secundaria Obligatoria. Datos similares encuentra el Plan Nacional sobre Drogas (PNsD) en la Encuesta sobre Uso de Drogas en Enseñanzas Secundarias en España (ESTUDES), que constata en su edición realizada en el curso escolar 2016-17 que el uso compulsivo de internet había crecido en nuestro país hasta el $21 \%$ entre los estudiantes de 14 a 18 años. Esto supone un 4,6\% más que en la anterior edición de ESTUDES realizada dos años antes (Plan Nacional sobre Drogas, 2018). Para medir este comportamiento se utilizó la escala CIUS (Compulsive Internet Use Scale) (Meerkerk, Van Den Eijnden, Vermulst y Garretsen, 2009).

Aunque se están desarrollando programas de prevención y tratamiento de las adicciones en internet que han mostrado cierta efectividad (Wölfling et al., 2019) aún escasean los programas disponibles ante la generalización de este fenómeno en nuestra sociedad. Debido a las importantes similitudes entre las conductas asociadas a los trastornos por uso de sustancias y el uso problemático o adictivo de internet, los autores de este trabajo hemos considerado adecuado desarrollar un programa de Cribado e Intervención Breve Digital vía web dirigido a la prevención y la reducción de los riesgos del uso problemático de Internet.

Este novedoso programa denominado PiensaTIC espera generalizar a este campo los resultados positivos obtenidos por esta metodología en el ámbito de los trastornos por uso de alcohol y otras sustancias.

Los objetivos de este programa preventivo y, por tanto, los resultados esperables de esta experiencia son de dos tipos. Por un lado, los cambios buscados en las actitudes y comportamientos de los estudiantes para reducir sus conductas de riesgo asociadas al UPI. Por otro, el conocimiento de las características del uso de internet y su nivel de uso problemático en el grupo de escolares que han participado en el programa. Respecto a los primeros, aún no podemos ofrecer resultados, ya que el diseño de la evaluación de este programa prevé que se realice una vez terminado el presente curso 2019-2020. Se presentan en el presente trabajo los resultados del segundo objetivo.

\section{Método}

\section{Participantes}

Los participantes en la primera edición piloto de este programa fueron los estudiantes pertenecientes a tres Institutos de Enseñanza Secundaria (IES) de la ciudad de Málaga que voluntariamente visitaron la web del programa y cumplimentaron todas las preguntas del cuestionario de su aplicación informática durante los meses de mayo y junio de 2019. 
En total lo realizaron 309 escolares con una edad comprendida entre los 12 y los 19 años, siendo el $49,51 \%$ chicos $(n=153)$ y el $51,49 \%$ chicas $(n=156)$, con edades entre 12 y 19 años $(M=14.99$ y $\mathrm{DT}=1,46$ ) y cursando en ese momento la ESO (Enseñanza Secundaria Obligatoria) y el primer curso de Bachillerato.

\section{Instrumentos}

Para valorar el uso problemático de internet se utilizaron dos escalas:

- Escala de Uso Compulsivo de Internet (Compulsive Internet Use Scale, CIUS; Meerkerk, Van Den Eijnden, Vermulst y Garretsen, 2009) que contiene 14 elementos que representan los elementos centrales del comportamiento compulsivo o adictivo clasificables en una escala Likert de 5 puntos, con una puntuación máxima de 56. CIUS ha mostrado buena estabilidad factorial en diferentes muestras de población y su consistencia interna es alta, demostrando una alta validez. Como punto de corte para la definición alto riesgo de uso compulsivo o problemático se ha consiserado, al igual que ESTUDES 2016-17 (Plan Nacional sobre Drogas, 2018), el punto de corte de 28 puntos.

- Cuestionario de Diagnostico de Young (Young's Diagnostic Questionnaire, YDQ; Young, 1998b) es un cuestionario breve con un formato de respuesta "si" o "no". Las ocho cuestiones del YDQ se basan en los criterios para el juego patológico en la cuarta edición del Manual diagnóstico y estadístico de los trastornos mentales DSM-IV (American Psychiatric Association, 2002): preocupación, tolerancia, pérdida de control, abstinencia, pérdida de relaciones u oportunidades, mentiras para evitar conflictos y afrontamiento disfuncional. En la aplicación de este instrumento está generalizada la puntuación de 5 o más criterios positivos como punto de corte para evaluar el uso problemático o adictivo de internet (Dowling \& Quirk, 2009).

Al comparar el YDQ con el CIUS existen coincidencias en la valoración de varios indicadores de conducta adictiva incluidos en ambos instrumentos, pero el YDQ incluye la valoración de algunos síntomas propios de las conductas adictivas como la tolerancia y la abstinencia que pueden señalar un nivel de trastorno más grave. Se considera que utilizando una combinación de estas escalas se recogen mejor los signos y síntomas del UPI que reflejan los criterios de diagnóstico para el trastorno por el uso de sustancias, el trastorno de juego patológico y el trastorno por juegos en internet. (Li, O’Brien, Snyder \& Howard, 2016).

El cuestionario de la aplicación web piensaTIC incluye, además, otras cuestiones diseñadas para valorar en cada estudiante:

- La autopercepción de normatividad de su comportamiento en internet en relación con su grupo de referencia (Gersh, Lee y McCarty, 2019).

- El estadio motivacional del cambio en el que se encuentra en la actualidad, su percepción de la importancia del mismo y su autoeficacia para afrontar dicho cambio. (DiClemente, Prochaska, Fairhurst, Velicer, Velasquez, Rossi, 1991).

\section{Procedimiento}

Para la primera edición del programa y para este trabajo se contactó con los orientadores educativos de tres IES de Málaga al objeto de conocer su opinión sobre la conveniencia de aplicar un programa de prevención del uso problemático de internet en sus respectivos centros escolares encontrando una excelente acogida a la propuesta en todos los casos. Estos profesionales facilitaron reuniones con los profesores tutores del centro, quienes dieron a conocer su opinión sobre la incidencia de este fenómeno en sus aulas y su disposición favorable a tratar el problema con sus estudiantes en sus espacios de tutoría, animándoles a visitar la página web www.piensaticplus.org y a cumplimentar el cuestionario de la aplicación desde sus ordenadores o sus teléfonos móviles

Al hacerlo, los estudiantes encontraban los siguientes contenidos administrados de forma automática por el algoritmo del programa informático de esta aplicación:

1. Autoevaluación de las actitudes y comportamientos de riesgo asociados a su uso de internet en las siguientes áreas: uso de redes sociales, uso de videojuegos, exposición a contenidos violentos y/o pornográficos, y juegos de azar y apuestas.

2. Informe preventivo personalizado según su nivel de riesgo (Bajo Riesgo: CIUS<19, Riesgo: CIUS $>18 Y<28$, Alto Riesgo: CIUS $=0>28$ y Muy Alto Riesgo: IDQ $=0>5$ ). 
3. Feedback sobre su percepción normativa del propio uso de internet en contraste con los datos sobre la incidencia y características de este uso en su grupo de referencia.

4. Información individualizada sobre los efectos y riesgos del uso problemático de internet y estrategias de reducción de sus riesgos personales en función de sus respuestas y su motivación para el cambio.

5. Oferta de asesoría on-line e información de recursos de ayuda para los casos que lo precisaran.

Además de estos contenidos dirigidos a los estudiantes, los educadores y familiares encuentran en el sitio web del programa información útil y contrastada sobre los riesgos asociados al uso problemático de Internet y las estrategias básicas para su reducción y prevención. De forma confidencial y con un código para cada centro los educadores pueden conocer en tiempo real el nivel de participación de los estudiantes y sus patrones de uso de internet, destacando la proporción de estudiantes por nivel de riesgo en cada centro.

\section{Resultados}

En relación con los contenidos en los que se implican en internet los escolares de nuestra muestra, se encuentra un uso frecuente, generalizado, y sin diferencia de género, de las redes sociales. Sí existen diferencias por género en cuanto a la participación en el resto de las actividades online: videojuegos (especialmente los de guerra en primera persona), páginas de adultos y juegos de apuestas. Todos estos usos son mucho más frecuentes entre los chicos que entre las chicas. Entre las mujeres solo una minoría refiere participar en videojuegos y muy pocas reconocen visitar páginas de adultos. Mientras que el $9,15 \%$ de los varones reconocen apostar dinero online con cierta habitualidad, entre las alumnas esta práctica es totalmente excepcional.

Tabla 1

Frecuencia de las diferentes actividades en internet.

\begin{tabular}{|c|c|c|c|}
\hline & $\%$ TODOS & $\% \mathrm{CHICOS}$ & $\%$ CHICAS \\
\hline \multicolumn{4}{|l|}{ Uso de las redes sociales } \\
\hline De 1 a 4 dias a la semana & 8,41 & 9,15 & 7,69 \\
\hline De 5 a 7 dias a la semana & 85,76 & 84,97 & 86,54 \\
\hline Varias veces a la semana & 94,17 & 94,12 & 94,23 \\
\hline \multicolumn{4}{|c|}{ Juegos de guerra o en primera persona } \\
\hline De 1 a 4 dias a la semana & 27,83 & 44,44 & 11,54 \\
\hline De 5 a 7 días a la semana & 20,71 & 35,29 & 6,41 \\
\hline Varias veces a la semana & 48,54 & $79,74(* *)$ & 17,95 \\
\hline \multicolumn{4}{|l|}{ Juegos de deportes o de habilidad } \\
\hline De 1 a 4 dias a la semana & 24,60 & 35,95 & 13,46 \\
\hline De 5 a 7 dias a la semana & 12,62 & 18,95 & 6,41 \\
\hline Varias veces a la semana & 37,22 & $54,90(* *)$ & 19,87 \\
\hline \multicolumn{4}{|l|}{ Páginas para adultos } \\
\hline De 1 a 3 dias al mes & 7,44 & 11,76 & 3,21 \\
\hline De 1 a 4 dias a la semana & 11,33 & 20,26 & 2,56 \\
\hline De 5 a 7 días a la semana & 16,18 & 30,72 & 1,92 \\
\hline Varias veces al mes o a la semana & 34,95 & $62,75(* *)$ & 4,49 \\
\hline \multicolumn{4}{|c|}{ Juego de dinero en juegos de casino o apuestas } \\
\hline De 1 a 3 días al mes & 1,94 & 3,92 & 0,00 \\
\hline De 1 a 4 días a la semana & 0,97 & 1,96 & 0,00 \\
\hline De 5 a 7 dias a la semana & 1,94 & 3,27 & 0,64 \\
\hline Varias veces al mes o a la semana & 4,85 & $9,15(* *)$ & 0,64 \\
\hline
\end{tabular}

${ }^{* *} p<.01$ según test chi cuadrado entre sexo y puntuación de cada ítem

El 25,57 \% de los participantes cumple el criterio de uso compulsivo de internet según la escala CIUS. No existen diferencias de importancia entre la proporción de chicos y chicas que cumplen este criterio según este instrumento de evaluación. Cuando usamos la escala YDQ para definir el uso problemático de internet, encontramos que un $20,06 \%$ de los participantes alcanza la puntuación que habitualmente se usa como punto de corte en esta escala: cinco síntomas o más de comportamiento adictivo. Aunque este resultado es un porcentaje total del alumnado muy cercano al que cumple el criterio de uso compulsivo según la escala CIUS, se observa, utilizando la escala YDQ, que un porcentaje significativamente mayor de varones que de mujeres pueden ser clasificados dentro de la categoría del uso problemático de internet. 
Tabla 2

Uso problemático de internet.

\begin{tabular}{lccc}
\hline & \% TODOS & \% CHICOS & \% CHICAS \\
\hline CIUS $=>28$ & 25,57 & 27,45 & 23,72 \\
YDQ $=>5$ & 20,06 & $25,49\left(^{(*)}\right.$ & 14,74 \\
\hline$* *$
\end{tabular}

${ }^{* *} p<.01$ según test chi cuadrado entre sexo y puntuación en cada escala

A continuación, se muestra la proporción de alumnos y alumnas que puntúan alto en cada ítem de la escala CIUS, encontrándose algunas diferencias significativas en cuanto a la frecuencia de la presentación de cada síntoma o consecuencia del comportamiento adictivo entre los chicos y las chicas.

Tabla 3

Porcentaje de alumnos que reconocen cumplir a menudo o muy frecuentemente los diferentes items de la escala CIUS

\begin{tabular}{|c|c|c|c|}
\hline & TODOS & CHICOS & CHICAS \\
\hline $\begin{array}{l}\text { CIUS } 1 \text { ¿Con qué frecuencia te ha resultado difícil dejar de } \\
\text { usar internet cuando estabas conectado? }\end{array}$ & 24,27 & 23,53 & 25,00 \\
\hline $\begin{array}{l}\text { CIUS } 2 \text { ¿Con qué frecuencia has seguido conectado a } \\
\text { internet a pesar de querer parar? }\end{array}$ & 26,86 & 28,10 & 26,14 \\
\hline $\begin{array}{l}\text { CIUS } 3 \text { ¿Con qué frecuencia tus padres, o amigos te dicen } \\
\text { que deberias pasar menos tiempo en internet? }\end{array}$ & 33,33 & $39,22\left(^{\star}\right)$ & 28,10 \\
\hline $\begin{array}{l}\text { CIUS } 4 \text { ¿Con qué frecuencia prefieres conectarte a internet } \\
\text { en vez de pasar el tiempo con otros (padres, amigos...)? }\end{array}$ & 10,36 & $11,76\left(^{\star \star}\right)$ & 8,97 \\
\hline $\begin{array}{l}\text { CIUS5 ¿Con qué frecuencia duermes menos por estar } \\
\text { conectado a internet? }\end{array}$ & 20,71 & 23,53 & 17,95 \\
\hline $\begin{array}{l}\text { CIUS } 6 \text { ¿Con qué frecuencia te encuentras pensando en } \\
\text { internet, aunque no estés conectado? }\end{array}$ & 9,71 & $10,46\left(^{*}\right)$ & 9,15 \\
\hline $\begin{array}{l}\text { CIUS } 7 \text { ¿Con qué frecuencia estás deseando conectarte a } \\
\text { internet? }\end{array}$ & 23,30 & 21,57 & 25.00 \\
\hline $\begin{array}{l}\text { ClUS } 8 \text { ¿Con qué frecuencia piensas que deberías usar } \\
\text { menos internet? }\end{array}$ & 19,74 & 15,69 & 23,72 \\
\hline $\begin{array}{l}\text { CIUS } 9 \text { ¿Con qué frecuencia has intentado pasar menos } \\
\text { tiempo conectado a internet y no lo has conseguido? }\end{array}$ & 10,68 & 7,84 & 13,73 \\
\hline $\begin{array}{l}\text { CIUS } 10 \text { ¿Con qué frecuencia intentas terminar tu trabajo a } \\
\text { toda prisa para conectarte a internet? }\end{array}$ & 15.53 & 16,99 & 14,10 \\
\hline $\begin{array}{l}\text { CIUS } 11 \text { ¿Con qué frecuencia descuidas tus obligaciones } \\
\text { (deberes, estar con la familia...) porque prefieres conectarte } \\
\text { a internet? }\end{array}$ & 15,21 & 15,03 & 15,38 \\
\hline $\begin{array}{l}\text { CIUS } 12 \text { ¿Con qué frecuencia te conectas a internet cuando } \\
\text { estas "de bajón"? }\end{array}$ & 42,39 & 39,87 & 44,87 \\
\hline $\begin{array}{l}\text { CIUS } 13 \text { ¿Con qué frecuencia te conectas a internet para } \\
\text { olvidar tus penas o sentimientos negativos? }\end{array}$ & 39,16 & 33,99 & $44,23\left({ }^{*}\right)$ \\
\hline $\begin{array}{l}\text { CIUS } 14 \text { ¿Con qué frecuencia te sientes inquieto, frustrado o } \\
\text { irritado si no puedes usar internet? }\end{array}$ & 11,65 & $12,42\left(^{\star}\right)$ & 10,90 \\
\hline
\end{tabular}

${ }^{\star} p<.05 y{ }^{\star \star} p<.01$ según test chi cuadrado entre sexo y puntuación de cada ítem

Ante la existencia de un número importante de alumnos y alumnas que muestran un claro riesgo de UPI, se valoró la disposición de los estudiantes de nuestra muestra a realizar cambios en su conducta, distribuyéndolos por estadios del cambio según sus contestaciones en el cuestionario (DiClemente et al, 1991). Aunque la mitad de los participantes no se plantea cambiar, la otra mitad se lo plantea en ocasiones, ha decidido hacer cambios en su uso de internet o lo está intentando.

Tabla 4

Motivación hacia el cambio.

\begin{tabular}{|c|c|c|c|}
\hline ESTADIOS DEL CAMBIO & $\%$ TODOS & $\% \mathrm{CHICOS}$ & $\%$ CHICAS \\
\hline $\begin{array}{l}\text { PRECONTEMPLACIÓN } \\
\text { No me estoy planteando cambiar mi forma de usar internet }\end{array}$ & 48,54 & 52,94 & 44,23 \\
\hline $\begin{array}{l}\text { CONTEMPLACIÓN } \\
\text { A veces pienso que me podria interesar usar internet de otra forma }\end{array}$ & 18,12 & 16,34 & 19,87 \\
\hline $\begin{array}{l}\text { PREPARACIÓN } \\
\text { He decidido cambiar en algo mi forma de usar internet }\end{array}$ & 11,65 & 13,07 & 10,26 \\
\hline $\begin{array}{l}\text { ACCIÓN } \\
\text { Estoy intentando cambiar mi forma de usar internet }\end{array}$ & 11,33 & 8,50 & 14,10 \\
\hline $\begin{array}{l}\text { MANTENIMIENTO } \\
\text { Hace tiempo que intenté y logré cambiar mi forma de usar internet }\end{array}$ & 7,12 & 7,19 & 7,05 \\
\hline $\begin{array}{l}\text { RECAIDA } \\
\text { Hace tiempo cambié mi forma de usar internet, pero después volví }\end{array}$ & 3,24 & 1,96 & 4,49 \\
\hline
\end{tabular}




\section{Discusión}

Dentro de las evidentes limitaciones de este estudio, los datos aportan algunos aspectos que señalan tendencias o circunstancias que pueden tener interés para su estudio en futuros trabajos con diseños más concluyentes.

En primer lugar, se puede señalar la coincidencia de datos del presente estudio con los de otros en cuanto a la generalización del uso recreativo de internet entre los estudiantes de enseñanza secundaria. También en el hecho de que diferentes usos de internet son mucho más frecuentes entre los alumnos que entre las alumnas (Durkee et al., 2012) (Fernández et al., 2015). Esta diferencia puede ser de importancia. Diferentes conductas online pueden suponer diferentes formas de desarrollo de un uso problemático de internet (Griffiths,M.D., Pontes, H.M.,2014).

Se encuentran, en un porcentaje apreciable del alumnado estudiado, signos y síntomas de un comportamiento problemático o adictivo. Utilizando para su detección la puntuación de 28 o más puntos en la escala CIUS, el Plan Nacional sobre Drogas encontró en su encuesta ESTUDES del curso 201617 que el $21 \%$ de los alumnos de 14 a 18 años hacían un uso compulsivo de internet, manteniéndose en el $20 \%$ en el curso 2018-19 (Plan Nacional sobre Drogas, 2019). Usando idéntico punto de corte de la misma escala encontramos que la incidencia del uso compulsivo o problemático en nuestra población es algo mayor (un 25,57 \%) pero claramente en la tendencia mostrada en dicha encuesta nacional. Aunque la última encuesta de ESTUDES muestra una diferencia entre la proporción de chicos y de chicas $(16,4 \%$ ellos y $23,4 \%$ ellas) que presentan uso compulsivo, no encontramos en este trabajo una diferencia significativa por género o, en todo caso, un mayor porcentaje de uso compulsivo entre los alumnos que entre las alumnas cuando se mide con este instrumento $(27,45 \%$ ellos y $23,72 \%$ ellas $)$.

Cuando se utiliza la escala YDQ para valorar este uso problemático o adictivo se encuentra un porcentaje de estudiantes que presenta UPI algo menor que usando el CIUS, pero no muy distinto (un $20 \%$ del total del grupo estudiado alcanza el punto de corte de 5 o más criterios positivos de dependencia propio de esta escala). Sin embargo, con este método de diagnostico las diferencias de género son bastante más evidentes, alcanzando el criterio establecido el $25,49 \%$ de los chicos frente al $14,74 \%$ de las chicas. Esta diferencia puede deberse a variables propias del género, a las diferentes conductas online mas extendidas entre un género y otro, o a una interacción de ambas variables.

Al estudiar la ocurrencia de los diferentes síntomas o consecuencias del comportamiento adictivo reflejados en las escalas utilizadas para diagnosticarlo en el grupo estudiado, se observa que el uso de internet como forma habitual de afrontar las emociones negativas es bastante común entre los participantes. Este uso de internet como intento de aliviar las emociones negativas está especialmente extendido entre las alumnas, ya que reconocen hacerlo a menudo, o muy frecuentemente, un 44,23 $\%$ de las mismas frente a un 33,29 \% de los alumnos. Más de la cuarta parte de los adolescentes independientemente de su género reconoce sentir con bastante frecuencia síntomas de pérdida de control sobre la duración de su uso de internet, alargando su tiempo de conexión o conectándose más frecuentemente de lo que pretendían. Una proporción similar, reconoce sentir con bastante frecuencia deseos de conectarse a internet sin existir tampoco diferencia de género en este síntoma adictivo. El $18,45 \%$ de la muestra reconoce síntomas de tolerancia y necesidad progresiva de incrementar su implicación en su uso de internet siendo este síntoma más común entre los alumnos $(21,57 \%)$ que entre las alumnas (15,38\%). Estos síntomas pueden ir asociados con cierta frecuencia a experimentar síntomas de tolerancia $(18,45 \%)$ y abstinencia cuando la conexión no es posible $(11,65 \%)$, ambos síntomas en mayor grado entre los chicos que entre las chicas. Las consecuencias del uso de internet reflejadas en conflictos con la familia y otras personas significativas son también detectables en mayor proporción entre los alumnos que entre las alumnas. Todos estos síntomas recuerdan en gran medida a los de los trastornos por uso de sustancias poniendo de manifiesto el nexo entre las adicciones a sustancias y las conductuales.

Ante un comportamiento problemático que implica la existencia de consecuencias negativas sufridas por los propios sujetos de estas conductas, surge la pregunta sobre la consciencia de perjuicio y el interés por cambiar, para eliminar o reducir los daños, en nuestra población. Utilizando el algoritmo de clasificación de estadios más común en el modelo transteórico del cambio (Díaz, 2001), encontramos que la mitad de la población estudiada se encuentra en el estadio de Precontemplación (estadio de cambio en el que los sujetos no valoran los eventuales perjuicios de su conducta y por tanto no se plantean en absoluto cambiarla). Pero casi un tercio de los participantes en nuestro estudio (un 29,77 $\%$ ) refieren encontrarse en los estadios de Contemplación (siendo en ocasiones conscientes de las consecuencias negativas de su comportamiento y por tanto ambivalentes ante el mismo) o Preparación para el cambio (teniendo decidido realizar cambios en un futuro muy próximo). Un $21,69 \%$ de los adolescentes estudiados se encuentra en Acción o Mantenimiento, por lo que está intentando cambiar, o 
ha cambiado ya, su forma de usar internet. Estos datos indican la existencia de un apreciable nivel de motivación para cambiar los eventuales usos problemático de internet por parte de los estudiantes de los IES en los que hemos intervenido y muestran una interesante ventana de oportunidad para seguir trabajando en el diseño y aplicación de intervenciones dirigidas a prevenir o a reducir los riesgos del uso de internet en esta población.

\section{Notas de autor}

La realización de este programa preventivo recibió una subvención para su diseño y desarrollo de la Consejería de Igualdad y Políticas Sociales de la Junta de Andalucía

\section{Referencias}

1. American Psychiatric Association (2002). Manual Diagnóstico y Estadístico de los Trastornos Mentales DSM-IV-TR. Barcelona: Masson.

2. Babor T.F., McRee B.G., Kassebaum P.A., Grimaldi P.L., Ahmed K. y Bray, J. (2007). Screening, Brief Intervention, and Referral to Treatment (SBIRT): toward a public health approach to the management of substance abuse. Substance Abuse, 28, 7-30. https://doi.org/10.1300/j465v28n03 03

3. Barata I.A., Shandro J.R., Montgomery M., Polansky R., Sachs C.J., Duber H.C., Weaver L.M., Heins A., Owen H.S., Josephson E.B., Macias-Konstantopoulos W. (2017). Effectiveness of SBIRT for Alcohol Use Disorders in the Emergency Department: A Systematic Review. The Western Journal Emergency Medicine, 18:1143-1152. https://doi.org/10.5811/westjem.2017.7.34373

4. Baumann S., Gaertner B., Haberecht K., Bischof G., John U., Freyer-Adam J. (2018) How alcohol use problem severity affects the outcome of brief intervention delivered in-person versus through computer-generated feedback letters. Drug Alcohol Dependendence, 183, 82-88. https:// doi.org/10.1016/j.drugalcdep.2017.10.032

5. $\quad$ Bewick B.M., West R., Gill J., O'May F., Mulhern B., Barkham M., Hill A.J. (2010). Providing web-based feedback and social norms information to reduce student alcohol intake: a multisite investigation. Journal of Medical Internet Research, 19, 12-59. https://doi.org/10.2196/jmir.1461

6. Borus J., Parhami I., Levy S. (2016). Screening, Brief Intervention, and Referral to Treatment. Child and Adolescent Psychiatric Clinics of North America, 25, 579-601. https://doi.org/10.1016/j. chc.2016.05.001

7. Boumparis N., Karyotaki E., Schaub M., Cuijpers P., Riper H. (2017). Internet interventions for adult illicit substance users: a Meta-analysis. Addiction, 112, 1521-1532. https://doi.org/10.1111/ add. 13819

8. Cronce J.M., Bittinger J.N., Liu J., Kilmer J.R. (2014). Electronic Feedback in College Student Drinking Prevention and Intervention. Alcohol Research: current reviews, 36, 47-62.

9. Derges J., Kidger J., Fox F., Campbell R., Kaner E., Hickman M. (2017). Alcohol screening and brief interventions for adults and young people in health and community-based settings: a qualitative systematic literature review. BMC Public Health, 9;17:562. https://doi.org/10.1186/ s12889-017-4476-4

10. Díaz J. (2001). El modelo de los estadios de cambio en la recuperación de las conductas adictivas. Escritos de Psicología, 5, 21-35.

11. DiClemente, C., Prochaska, J.O., Fairhurst, S.K., Velicer, W. F., Velasquez, M., Rossi, J.S. (1991). The process of smoking cessation: An analysis of precontemplation, contemplation, and preparation stages of change. Journal of Consulting and Clinical Psychology, 59, 295-304. https://doi. org/10.1037/0022-006x.59.2.295

12. Dimeff L.A., Baer J.S., Kivlahan D.R., Marlatt G.A. (1999). Brief Alcohol Screening and Intervention for College Students. New York: Guilford Press.

13. Donoghue K., Patton R., Phillips T., Deluca P., Drummond C., (2014). The Effectiveness of Electronic Screening and Brief Intervention for Reducing Levels of Alcohol Consumption: A Systematic Review and Meta-Analysis. Journal Medicine Internet Research, 16, e142. https://doi. org/10.2196/jmir.3193

14. Dowling NA, Quirk KL. (2009). Screening for Internet dependence: do the proposed diagnostic criteria differentiate normal from dependent Internet use? Cyberpsychology Behavior, 12, 21-27. https://doi.org/10.1089/cpb.2008.0162 
15. Durkee T., Kaess M., Carli V., Parzer P., Wasserman C. Floderus B., Wasserman D. (2012). Prevalence of pathological internet use among adolescents in Europe: demographic and social factor. Addiction, 07, 2210- 2222. https://doi.org/10.1111/j.1360-0443.2012.03946.x

16. Duroy D., Boutron I., Baron G., Ravaud P., Estellat C., Lejoyeux M. (2016). Impact of a computer-assisted Screening, Brief Intervention and Referral to Treatment on reducing alcohol consumption among patients with hazardous drinking disorder in hospital emergency departments. The randomized BREVALCO trial. Drug and Alcohol Dependence,1, 236-44. https://doi. org/10.1016/j.drugalcdep.2016.06.018

17. Fernández T., Alguacil J., Almaraz A., Cancela J.M., Delgado M., García M., Jiménez E., Llorca J., Molina A.J., Ortíz R., Valero L.F., Martín V. (2015). Uso problemático de internet en estudiantes universitarios: factores asociados y diferencias de género, Adicciones, 27, 265-275. https://doi. org/10.20882/adicciones.751

18. Fraeyman J., Van Royen P., Vriesacker B., De Mey L., Van Hal G. (2012) How is an electronic screening and brief intervention tool on alcohol use received in a student population? A qualitative and quantitative evaluation. Journal of medical Internet research, 14, e56. https://doi.org/10.2196/ imir.1869

19. Ganz T., Braun M., Laging M., Schermelleh-Engel K., Michalak J., Heidenreich T. (2018). Effects of a stand-alone web-based electronic screening and brief intervention targeting alcohol use in university students of legal drinking age: A randomized controlled trial. Addictive Behaviors. 77:81-88. https://doi.org/10.1016/j.addbeh.2017.09.017

20. Gersh E., Lee C.M., McCarty C.A. (2019). Changes in peer norms as a mediator of reduction in adolescent alcohol use. Sustance use \& misuse, 54, 1611-1617. https://doi.org/10.1080/108260 $\underline{84.2019 .1594906}$

21. Goldberg, I. (1996). Internet Addiction Support Group. Recuperado de http://users.rider.edu/ suler/psycyber/supportgp.html.

22. Gómez, P., Rial, A., Braña, T., Varela, J., y Barreiro, C. (2014). Evaluation and early detection of problematic Internet use in adolescents. Psicothema, 26, 21-26. https://doi.org/10.7334/psicothema2013.109

23. Greenfield D.N (1999).Psychological characteristics of compulsive internet use: a preliminary analysis. Cyberpsychol \& Behavior, 2, 403-4012. https://doi.org/10.1089/cpb.1999.2.403

24. Griffiths, M.D \& Pontes, H.M. (2014). Internet Addiction Disorder and Internet Gaming Disorder are Not the Same. Addiction Research \& Therapy, 5, e124. https://doi.org/10.4172/21556105.1000e124

25. Guertler D., RumpfH.J., Bischof A., Kastirke N., Petersen K.V., John U., Meyer C. (2014). Assessment of Problematic Internet Use by the Compulsive Internet Use Scale and the Internet Addiction Test: A Sample of Problematic and Pathological Gamblers. European Addiction Research; 20, 75-81. https://doi.org/10.1159/000355076

26. Hallett J., Maycock B., Kypri K., Howat P., McManus A. (2009). Development of a Web-based alcohol intervention for university students: processes and challenges. Drug and Alcohol Review, 28, 31-39. https://doi.org/10.1111/j.1465-3362.2008.00008.x

27. Hargraves D., White C., Frederick R., Cinibulk M., Peters M., Young A., Elder N. (2017). Implementing SBIRT (Screening, Brief Intervention and Referral to Treatment) in primary care: lessons learned from a multi-practice evaluation portfolio. Public Health Reviews, 38:31. https://doi. org/10.1186/s40985-017-0077-0

28. Harris S.K., Louis-Jacques J., Knight J.R. (2014). Screening and brief intervention for alcohol and other abuse. Adolescent Medicine: State of the Art Reviews, 25, 126-56.

29. Hong S., Zalesky A., Cocchi L., Fornito A., Choi E., Kim H., Suh J., Kim C., Kim J.W., Yi S. H. (2013). Decreased Functional Brain Connectivity in Adolescents with Internet Addiction. Plos One, 8, e57831. https://doi.org/10.1371/journal.pone.0057831

30. Kypri K., Langley J.D., Saunders J.B., Cashell-Smith M.L. (2008). Herbison P. Randomized controlled trial of web-based alcohol screening and brief intervention in primary care. Archives of Internal Medicine, 168, 530-536. https://doi.org/10.1001/archinternmed.2007.109

31. Landy M.S., Davey C.J., Quintero D., Pecora A., McShane K.E. (2016). A systematic review of the effectiveness of brief interventions for alcohol abuse among adults in emergency departments. Journal of Substance Abuse Treatment. febrer; 61: 1-12. https://doi.org/10.1016/j.jsat.2015.08.004 
32. Li W.,O'Brien J.E.,Snyder S.M.,Howard M. O. (2016). Diagnostic Criteria for Problematic Internet Use among U.S. University Students: A Mixed-Methods Evaluation. PLoS One. Jan 11;11:e0145981. https://doi.org/10.1371/journal.pone.0145981

33. Lin F., Zhou Y., Du Y., Qin L., Zhao Z., Xu J., Lei H. (2012). Abnormal White Matter Integrity in Adolescents with Internet Addiction Disorder: A Tract-Based Spatial Statistics Study. Plos One, 7, e30253. https://doi.org/10.1371/journal.pone.0030253

34. Meerkerk G.J., Van Den Eijnden R.J., Vermulst A.A., Garretsen H.F., (2009) The Compulsive Internet Use Scale (CIUS): some psychometric properties. Cyberpsychology and Behavior 12:16. https://doi.org/10.1089/cpb.2008.0181

35. Morahan-Martin J.M. (2004). How internet users find, evaluate, and use online health information: a cross-cultural review. Cyberpsychology Behaviors, 7, 497-510. https://doi.org/10.1089/ cpb.2004.7.497

36. Muñoz R., Ortega R.,Batalla C., LópezM.R., Manresab J.M., Torán P. (2014). Acceso y uso de nuevas tecnologías entre los jóvenes de educación secundaria, implicaciones en salud. Estudio JOITIC. Atención Primaria, 46, 77-88. https://doi.org/10.1016/i.aprim.2013.06.001

37. Pedersen E.R., Grow J., Duncan S., Neighbors C., Larimer M.E. (2012). Concurrent validity of an online version of the Timeline Followback assessment. Psychology of addictive behaviors: Journal of the Society of Psychologist in addictive Behaviors, 26, 672-677 https://doi.org/10.1037/ a0027945

38. Plan Nacional sobre Drogas (2018) ESTUDES 2016-17 Recuperado de $h t t p: / / w w w . p n s d . m s c b s$. gob.es/profesionales/sistemas/nformacion/sistemalnformacion/pdf/2016 2017 ESTUDES.pdf.

39. Plan Nacional sobre Drogas (2019) ESTUDES 2018-19 Recuperado de http://www.pnsd. mscbs.gob.es/profesionales/sistemasInformacion/sistemalnformacion/pdf/ESTUDES_2018-19 Informe.pdf.

40. Puerta-Cortésa D. X., Carbonell X. y Chamarro A. (2012). Análisis de las propiedades psicométricas de la versión en español del Internet Addiction Test. Trastornos Adictivos. 14, 99-104. https:// doi.org/10.1016/s1575-0973(12)70052-1

41. Secades-Villa R., Calafat A., Fernández-Hermida J.R., Juan M., Duch M., Skärstrand E., Becoña E. y Sanela Talic S. (2014). Tiempo de uso de Internet y efectos psicosociales adversos en adolescentes europeos. Adicciones, 26, 247-253. https://doi.org/10.20882/adicciones.6

42. Shapira N.A., Lessig M.C., Goldsmith T.D., Szabo S.T., Lazoritz M., Gold M.S., Stein D.J. (2003). Problematic internet use: proposed classification and diagnostic criteria. Depression and Anxiety, 17, 207-216. https://doi.org/10.1002/da.10094

43. Spada M.M. (2014). An overview of problematic Internet use. Addictive Behaviors, 39, 3-6. https:// doi.org/10.1016/j.addbeh.2013.09.007

44. Tansil K.A., Esser M.B., Sandhu P., Reynolds J.A., Elder R.W., Williamson R.S., Chattopadhyay S.K., Bohm M.K., Brewer R.D., McKnight-Eily L.R., Hungerford D.W., Toomey T.L., Hingson R.W., Fielding J.E. y Community Preventive Services Task Force. (2016a). Alcohol Electronic Screening and Brief Intervention: A Community Guide Systematic Review. American Journal Preventiva Medicine, 51, 801-811. https://doi.org/10.1016/j.amepre.2016.04.013

45. Tansil K.A., Esser M.B., Sandhu P., Reynolds J.A., Elder R.W., Williamson R.S., Chattopadhyay S.K., Bohm M.K., Brewer R.D., McKnight-Eily L.R., Hungerford D.W., Toomey T.L., Hingson R.W., Fielding J.E. y Community Preventive Services Task Force. (2016b). Alcohol Electronic Screening and Brief Intervention: Recommendation of the Community Preventive Services Task Force. American Journal of Preventive Medicine, 51, 812-813. https://doi.org/10.1016/j.amepre.2016.04.012

46. Tsitsika A., Janikian M., Schoenmakers T.M., Tzavela C.E., Olafsson K., Wójcik S., Macarie G.F., Tzavara C., Richardson C. (2014). Internet addictive behavior in adolescence: a cross-sectional study in seven European countries. Cyberpsychology, Behavior, and Social Networking, 17, 52835. https://doi.org/10.1089/cyber.2013.0382

47. Yellowlees P.M., Marks S. (2007). Problematic Internet use or Internet addiction? Journal Computers in Human Behavior, 23, 1447-1453. https://doi.org/10.1016/j.chb.2005.05.004

48. Young K. S. (1998a). Caught in the net. New York: John Wiley \& Sons.

49. Young, K. S. (1998b). Internet addiction: The emergence of a new clinical disorder. Cybersychology \& Behavior, 1, 237-244. https://doi.org/10.1089/cpb.1998.1.237

50. Walton M.A., Bohnert K., Resko S., Barry K.L., Chermack S.T., Zucker R.A., Zimmerman M.A., Booth B.M., Blow F.C. (2013). Computer and therapist based brief interventions among cannabis-using adolescents presenting to primary care: one year outcomes. Drug and Alcohol Dependence, 132, 646-653. https://doi.org/10.1016/j.drugalcdep.2013.04.020 
51. Wright T.E. (2017). Screening, brief intervention, and referral to treatment for opioid and other substance use during infertility treatment. Fertility and Sterility, 108, 214-221. https://doi.org/10.1016/j. fertnstert.2017.06.012

52. Wölfling K.,Müller K., Dreier M., Ruckes C., Deuster O., Batra A., Mann K., Musalek M., Schuster A., Lemenager T, Hanke S., Beutel M. (2019). Efficacy of Short-term Treatment of Internet and Computer Game Addiction A Randomized Clinical Trial. JAMA Psychiatry, 76, 1018-1025. https:// doi.org/10.1001/jamapsychiatry.2019.1676

RECIBIDO: 21 de noviembre de 2019 MODIFICADO: 2 de junio de 2020 ACEPTADO: 22 de junio de 2020 